\title{
Predicting renal outcomes in immunoglobulin A vasculitis nephritis; from ISKDC classification to Oxford MEST-C classification
}

\author{
Mohsen Akhavan Sepahi ${ }^{\left.{ }^{*} \mathbb{(}\right)}$, Muhammed Mubarak $^{2}{ }^{\circledR}$, Steven James Kellner $^{3}$ \\ ${ }^{1}$ Department of Pediatric Nephrology, School of Medicine and Pediatric Clinical Research of Development Center, Qom University of \\ Medical Sciences, Qom, Iran

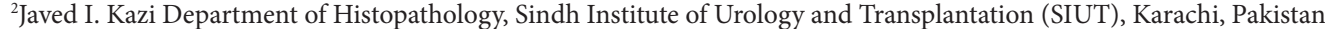 \\ ${ }^{3}$ Head International Research and Development, Mesencell Biotech International Ltd, 20-22 Wenlock Road, London, N1 7GU, United \\ Kingdom
}

\section{ART I C L E IN F O}

Article Type:

Review

\section{Article History:}

Received: 30 October 2019

Accepted: 2 December 2019

Published online: 14 December 2019

\section{Keywords:}

Henoch-Schönlein purpura nephritis, Immunoglobulin A vasculitis nephritis, Immunoglobulin A nephropathy, IgA vasculitis, Extracapillary proliferation, IgA nephropathy, Oxford classification

\begin{abstract}
A B S T R A C T
Immunoglobulin A vasculitis nephritis (IgAVN) or Henoch-Schönlein purpura nephritis (HSPN) is the most common type of secondary $\operatorname{IgA}$ nephropathy (IgAN), particularly in children. Overall, its prognosis is good, but in a significant number of cases, it does lead to chronic kidney disease (CKD) and end-stage renal disease (ESRD). Various classifications exist to predict the long-term outcome of kidney disease in these diseases, but none is universally accepted. We searched PubMed, Web of Science Embase, EBSCO, Scopus and directory of open access journals (DOAJ) with keywords of Henoch-Schönlein purpura nephritis, immunoglobulin A vasculitis nephritis, immunoglobulin A nephropathy, IgA vasculitis, extracapillary proliferation, IgA nephropathy, leukocytoclastic vasculitis, endstage renal disease, Oxford classification, crescent and childhood IgA vasculitis. IgAVN in children presents most often with crescents and endocapillary proliferation, with relapsing and remitting course clinically. Due to morphological resemblance of IgAVN and IgAN, the Oxford MEST-C scores could be applied for determining the long-term outcomes in the former disease. A critical concern in applying Oxford classification for IgAVN is that limited number of children with IgAVN exists in any one center and also a relatively short period of follow-up. Hence, further work in this regard is necessary. Preliminary evidence suggests that Oxford MEST-C classification is valid in predicting long-term kidney outcomes in children with IgAVN and the classification can also be used in adults. However, further, large scale, multicenter, international collaborative studies are needed to address the unmet issues.
\end{abstract}

Implication for health policy/practice/research/medical education:

It is possible to use Oxford MEST-C scores for predicting the long-term outcomes in IgAVN; however, further work is necessary to demonstrate the full validation of morphologic variables of Oxford MEST-C classification in this disease.

Please cite this paper as: Akhavan Sepahi M, Mubarak M, Kellner JS. Predicting renal outcomes in immunoglobulin A vasculitis nephritis; from ISKDC classification to Oxford MEST-C classification. J Renal Inj Prev. 2020; 9(1): e08. doi: 10.15171/jrip.2020.08.

\section{Introduction}

Immunoglobulin A vasculitis nephritis (IgAVN) or Henoch-Schönlein purpura nephritis (HSPN) is the most common type of secondary $\operatorname{IgA}$ nephropathy (IgAN) $(1,2)$. It is the most common form of vasculitis in children. In addition, it is not uncommon in adults, too $(3,4)$. The annual incidence of Immunoglobulin A vasculitis (IgAV) or Henoch-Schönlein purpura (HSP) is assessed at between 6.1 and 26.7 for each 100000 of children $(5,6)$. The data suggests that HSP is more common in children of Asian origin than in white or black children (1-5). HSP, appears most frequently in children between the ages of three and
10 years, with the peak age at onset ranging between 4 and 6 years (1-5). Considering that the occurrence of IgAVN differs with age, a bimodal distribution of disease with peaks at 20-29 years and 60 to 69 years in adults has been detected (3,7-10).

\section{Materials and Methods}

For this review, we searched some of sources in the English language including PubMed, Web of Science Embase, EBSCO, Scopus and directory of open access journals (DOAJ). The search was directed toward using combinations of the following keywords and/or their 
equivalents; Henoch-Schönlein purpura nephritis, immunoglobulin A vasculitis nephritis, immunoglobulin A nephropathy, IgA vasculitis, extracapillary proliferation, IgA nephropathy, leukocytoclastic vasculitis, endstage renal disease, Oxford classification, crescent and childhood $\operatorname{Ig}$ A vasculitis.

\section{Results}

A total of 35 research and review articles relevant to this topic, either directly or indirectly, were found. These were scrutinized in detail and relevant information was gathered and synthesized into this brief review.

\section{Discussion}

The clinical presentation of IgAV comprises a tetrad of gastrointestinal tract involvement, cutaneous purpura, joint disease and renal involvement. However, the pattern of presentation of the disease depends largely on the age of the individuals. Compared to children, adults present with systemic involvement more commonly, with notable risk of chronic kidney disease (CKD) and bowel ischemia (11). IgAV is a systemic vasculitis (leukocytoclastic vasculitis) affecting the small vessels and is characterized by the deposition of IgA-containing immune complexes, particularly in the kidneys, skin and other organs. IgAVN and IgAN are believed to be related diseases, since both possibly share a related immunopathogenic mechanism which engages abnormally glycosylated $\operatorname{IgA1}(12,13)$. IgAVN tends to display itself as an acute proliferative glomerulonephritis, while IgAN tends to have an indolent course. Both of the diseases characteristically present concurrently with an upper respiratory infection (synpharygitic glomerulonephritis) or within a few days after the infection (14). This is in contrast to postinfectious acute glomerulonephritis, which presents at least two weeks after infection. IgAN, usually presents as a slowly progressive disease most of the time; however, it progresses to end-stage renal disease (ESRD) in a significant number of affected individuals. In general, kidney lesions in IgAVN range from minimal change lesions to focal or diffuse proliferative glomerulonephritis and extra-capillary proliferation. Immunofluorescence (IF) studies, on the other hand, are consistent and classically show dominant or co-dominant IgA deposition in the mesangial areas (2-6). Importantly, kidney involvement occurs more commonly in adults than in children $(15,16)$.

IgAVN is classically a self-limiting disease and the prognosis is mainly good. However, kidney disease may progress rendering the kidney involvement as the most important prognostic parameter. Recent studies have observed that the long-term outcome of IgAV largely depends on the severity of kidney damage. Studies show that nephritis occurs in $45 \%-85 \%$ of adults with IgAV; however, it mostly runs a benign course. Long-term prognosis of IgAV is related to the intensity and severity of nephritis (15-19). IgAVN shares a similar kidney pathology spectrum as IgAN, albeit with mild differences. It is often considered as part of the morphological spectrum of IgAN. Despite the morphologic and pathogenetic relationships among the two diseases, these have traditionally been classified by two separate methods of Oxford classification for IgAN and International Study of Kidney Disease in Children (ISKDC) classification for $\operatorname{IgAVN}(20,21)$. Among these, the classification of ISKDC is fundamentally based on the presence and extent of extra-capillary proliferation (crescents), while crescents were given less importance in the original Oxford classification for $\operatorname{IgAN}(22,23)$. IgAVN is characterized by more endocapillary hyper-cellularity, extracapillary proliferation (crescents), fibrin deposits and glomerular IgA deposits, principally along the capillary basement membranes (24). Crescents are more commonly detected in kidney biopsies of IgAVN patients. However, the significance of crescents in determining the long-term outcome of $\operatorname{IgAV}$ is still under investigation. Many investigators have found that a high proportion of crescents portends adverse kidney consequences; in contrast, other studies did not show the prognostic significance of crescents. Extracapillary proliferation (crescent formation) is accompanied by fibrinoid necrosis, capillary wall destruction and endocapillary hypercellularity and polymorphonuclear neutrophil infiltration, not only in the glomeruli but also the extraglomerular vessels. The long-term outcome of individuals with kidney involvement of varying severity has not been fully described. Renal outcome is largely good in patients with minimal involvement and the disease is self-limited; however, some patients with persistent kidney involvement, particularly crescentic glomerulonephritis on renal biopsy, may develop ESRD subsequently in their life. Therefore, prompt and precise detection and characterization of renal involvement is a key challenge for optimal treatment selection and followup. Hence, a renal biopsy and morphological classification has a crucial role to establish the diagnosis, prognosis and optimal management of disease. Accordingly, there is a need for a reliable, feasible and proven classification to help clinicians more accurately assess the treatment modalities for patients with IgAVN. However, the value of renal biopsy as a predictor of the outcome is dependent on which morphologic lesions harbinger prognostic implications. The ISKDC classifies IgAVN as; 1) minimal glomerular abnormalities 2) mesangial proliferation, 3) mesangial proliferation with extracapillary proliferation in less than $50 \%$ of the glomeruli, 4) mesangial proliferation with extracapillary proliferation in $50 \%-75 \%$ of the glomeruli, and 5) mesangial proliferation with extracapillary proliferation in more than $75 \%$ of the glomeruli, and finally 6) membranoproliferative-like lesions (25). It is important to remember that; this classification is mostly dedicated to the morphologic features of glomeruli and only reflects active inflammation and does not address 
chronic changes of the glomeruli or pathologic lesions of vessels and tubulointerstitium. For example, it was shown that infiltration of inflammatory cells, including macrophages and neutrophils in the interstitium, mostly macrophage infiltration, commonly detected in tubuleinterstitial diseases, may have a prognostic significance. Accordingly, the intensity of macrophage infiltration has been shown to be related to the intensity of glomerular injury too (26-30). The original Oxford classification of IgAN, covered four morphologic aspects of mesangial hypercellularity (mesangial proliferation) score (M); segmental glomerulosclerosis (S), endocapillary hypercellularity (E) and tubular atrophy/interstitial fibrosis (T), and was named as MEST score. These morphologic variables were shown to be the independent prognosticators of clinical outcome. Patients with IgAVN were excluded from the analysis during the formulation of the OxfordMEST classification. Since, crescents (extracapillary hypercellularity) were not incorporated in the original Oxford classification, subsequent to the publication of the Oxford-MEST classification, various investigations were conducted, which highlighted the significance of crescents (31). Accordingly, the scoring of crescents was also added as an Oxford classification parameter and a factor of predictive value resulting in the modification of MEST score to the MEST-C scores (32). Extracapillary proliferation signifies a serious form of glomerular damage in which leukocyte infiltration and cellular proliferation lead to capillary wall damage and rupture due to fibrinoid necrosis (33). The rupture of the glomerular basement membranes leads to leakage of fibrin and inflammatory cells in Bowman's space and the proliferation of visceral and parietal epithelial cells, intermixed with mononuclear leukocytes in the urinary space. This condition is frequently linked with endocapillary proliferation and infiltration of inflammatory cells, including macrophages and neutrophils both within the endocapillary compartment and extracapillary compartment (glomerular urinary space). In contrast to the more gradual progressive nature of IgAN, IgAV in children presents more often with crescents and endocapillary proliferation, with remitting and relapsing course. In fact, glomerular morphologic lesions in IgAV tend to present as an acute glomerular inflammatory pattern involving both endocapillary and extracapillary compartments, while IgAN tends to present as slow and progressive mesangiopathic pattern of injury. The risk of development of CKD in individuals with IgAVN ranges from $5 \%$ to $20 \%$ in children and approaches $30 \%$ in adults. Given that various clinical and laboratory parameters, like the quantity of proteinuria, deranged kidney function, and presence of hypertension at initial admission, have been detected as risk factors for CKD in IgAVN, the morphologic lesions of the glomeruli and the tubulointerstitial compartment and also of vessels, would also be of great importance as prognostic parameters in clinical practice (30-34). Additionally, morphologic lesions on histology are a principal feature to determine the use of immunosuppressive therapy. Like lupus nephritis, IgAVN, is also an inflammatory kidney disease, and in lupus nephritis, the treatment modalities are selected according to the histological classification, the same might apply to IgAVN. In view of the morphological resemblances of IgAVN and IgAN, the Oxford MEST-C scores could be applied for predicting the long-term outcomes in $\operatorname{IgAVN}(21,32,35,36)$. A critical concern in applying this classification for IgAVN is that limited number of children with IgAVN exist at any single center and a relatively short period of follow-up. Hence, the morphologic variables of Oxford MEST-C classification have not been validated enough in children. Furthermore, the IgAN Network (IgANN) working group does not advocate application of Oxford MEST-C score in IgAVN, since their cases did not include IgAV cases and exclusively included IgAN cases (31-34). Hence, with the latest update to the Oxford score, further studies on the clinical significance of the updated components in IgAVN are warranted, together with other currently used classifications (31-36). Recently, to detect the usefulness of the Oxford MEST-C classification in children with IgAVN, 58 male and 46 female children (104 children) aged 4-17 years (median age of 10 years) were studied. The study showed, mesangial proliferation (M1) was significantly associated with tubular atrophy/interstitial fibrosis (T score) and proteinuria. Moreover, crescents in more than $25 \%$ of glomeruli (C2) were associated with reduced eGFR (estimated glomerular filtration rate) at the time of biopsy (37). This study showed that patients with endocapillary proliferation (E1), mesangial proliferation (M1), segmentalglomerulosclerosis (S1) and extracapillary proliferation ( $\mathrm{C} 1$ and $\mathrm{C} 2$ ) were more likely to respond to high-dose methylprednisolone. This study showed that $\mathrm{S}$ lesion was significantly associated with primary outcome, and tubular atrophy/interstitial fibrosis (T) was significantly and inversely associated with the remission of proteinuria and also clinical remission. They concluded that Oxford MEST-C is valid for children with IgAVN. They also concluded that $S$ and $T$ scores, which are ignored in the ISKDC classification, can be applied to evaluate kidney outcomes of IgAVN (37).

\section{Conclusion}

It is possible to apply Oxford MEST-C scores to determine the long-term outcomes in IgAVN. However, a critical concern in using Oxford classification for $\operatorname{IgAVN}$, is the small number of children with this disease at any one center and a relatively short period of follow-up. Therefore, further work in this regard is necessary.

Authors' contribution

MAS and MM searched the data and prepared the draft of the manuscript. MM edited the manuscript too. SJK made 
some critical comments on the manuscript. MAS and MM conducted the final check, final editing and finalized the paper. All authors read and signed the final manuscript.

\section{Conflicts of interest}

The authors declared no competing interests.

\section{Ethical considerations}

Ethical issues (including plagiarism, data fabrication, double publication) have been completely observed by the authors.

\section{Funding/Support}

None.

\section{References}

1. Jennette JC. Overview of the 2012 revised International Chapel Hill Consensus Conference nomenclature of vasculitides. Clin Exp Nephrol. 2013;17:603-6. doi: 10.1007/ s10157-013-0869-6.

2. Davin JC, Coppo R. Henoch-Schönlein purpura nephritis in children. Nat Rev Nephrol. 2014;10:563-73.

3. Ueda Hi, Miyazaki Y, Tsuboi N, Hirano K, Yokote S, Kobayashi E, et al. Clinical and Pathological Characteristics of Elderly Japanese Patients with IgA Vasculitis with Nephritis: A Case Series. Intern Med. 2019;58:31-8. doi: 10.2169/internalmedicine.1379-18.

4. Sepahi MA, Shajari A, Shakiba M, Shooshtary FK, Salimi MH. Acute glomerulonephritis: a 7 years follow up of children in center of Iran. Acta Med Iran. 2011;49(6):375-8.

5. Mir S, Yavascan O, Mutlubas F, Yeniay B, Sonmez F. Clinical outcome in children with Henoch-Schönlein nephritis. Pediatr Nephrol. 2007;22:64-70

6. Saulsbury FT. Clinical update: Henoch-Schönlein purpura. Lancet. 2007;369:976-8.

7. Coppo R, Andrulli S, Amore A, Gianoglio B, Conti G, Peruzzi L, et al. Predictors of outcome in HenochSchönlein nephritis in children and adults. Am J Kidney Dis. 2006;47:993-1003.

8. Ronkainen J, Nuutinen M, Koskimies O.The adult kidney 24 years after childhood Henoch-Schönlein purpura: a retrospective cohort study. Lancet. 2002;360:666-70.

9. Kawasaki Y, Suzuki J, Sakai N, Nemoto K, Nozawa R, Suzuki S, et al. Clinical and pathological features of children with Henoch-Schoenlein purpura nephritis: risk factors associated with poor prognosis. Clin Nephrol. 2003;60:15360 .

10. Shajari H, Shajari A, Sepahi MA, Mehrparvar AH, Roghani $\mathrm{R}$, Nakhaei $\mathrm{MH}$. Relationship between arterial blood pressure and body mass index of school age children of southern region of Iran. Acta Med Iran. 2011;49:737-41.

11. González-Gay MA, López-Mejías R, Pina T, Blanco R, Castañeda S. IgA vasculitis: genetics and clinical and therapeutic management. Curr Rheumatol Rep. 2018;20:24. doi: 10.1007/s11926-018-0735-3.

12. Nicoara O, Twombley K. Immunoglobulin a nephropathy and immunoglobulin a vasculitis. Pediatr Clin North Am. 2019; 66:101-10. doi: 10.1016/j.pcl.2018.08.008.

13. Ozen S, Marks SD, Brogan P, Groot N, de Graeff N, Avcin
T, et al. European consensus-based recommendations for diagnosis and treatment of immunoglobulin A vasculitisthe SHARE initiative. Rheumatology. 2019;58:1607-1616. doi: 10.1093/rheumatology/kez041.

14. Guo CP, Lu C. Risk Factors for Renal Involvement in Patients with Immunoglobulin A Vasculitis/Henoch-Schönlein Purpura: An Updated Review. Int J Dermatol Venereol. 2019;2:84-8. doi: 10.1097/01.JD9.0000559510.85685.c9.

15. Kuret T, Lakota K, Žigon P, Ogrič M, Sodin-Šemrl S, Čučnik $S$, et al. Insight into inflammatory cell and cytokine profiles in adult IgA vasculitis. Clin Rheumatol. 2019;38:331-8. doi: 10.1007/s10067-018-4234-8.

16. Berthelot L, Jamin A, Viglietti D, Chemouny JM, Ayari $\mathrm{H}$, Pierre $\mathrm{M}$, et al. Value of biomarkers for predicting immunoglobulin A vasculitis nephritis outcome in an adult prospective cohort. Nephrol Dial Transplant. 2017;33:157990. doi:10.1093/ndt/gfx300.

17. Lu S, Liu D, Xiao J, Yuan W, Wang X, Zhang X, et al. Comparison between adults and children with HenochSchönlein purpura nephritis. Pediatr Nephrol. 2015; 30:791-6.

18. Pillebout E, Thervet E, Hill G, Alberti C, Vanhille P, Nochy D. Henoch-Schönlein Purpura in adults: outcome and prognostic factors. J Am Soc Nephrol. 2002;13:1271-1278.

19. Goldstein AR, White RH, Akuse R, Chantler C. Longterm follow-up of childhood Henoch-Schönlein nephritis. Lancet. 1992;339:280-282.

20. Cattran DC, Coppo R, Cook HT, Feehally J, Roberts IS, Troyanov S, et al. The Oxford classification of IgA nephropathy: rationale, clinicopathological correlations, and classification. Kidney Int. 2009;76:534-45.

21. Barbour SJ, Espino-Hernandez G, Reich HN, Coppo R, Roberts IS, Feehally J, et al. The MEST score provides earlier risk prediction in $\lg$ A nephropathy. Kidney Int. 2016;89:167-75.

22. Halling SF, Söderberg MP, Berg UB. Henoch Schönlein nephritis: clinical findings related to renal function and morphology. Pediatr Nephrol. 2005;20:46-51.

23. Huang X, Ma L, Ren P, Wang H, Chen L, Han H, et al. Updated Oxford classification and the international study of kidney disease in children classification: application in predicting outcome of Henoch-Schönlein purpura nephritis. Diagn Pathol. 2019;14:40. Doi: 10.1186/s13000019-0818-0.

24. Kim CH, Lim BJ, Bae YS, Kwon YE, Kim YL, Nam KH, et al. Using the Oxford classification of IgA nephropathy to predict long-term outcomes of Henoch-Schönlein purpura nephritis in adults. Mod Pathol. 2014;27:972-82.

25. 25.Yang $\mathrm{YH}, \mathrm{Yu} \mathrm{HH}$, Chiang BL. The diagnosis and classification of Henoch-Schönlein purpura: an updated review. Autoimmun Rev. 2014;13:355-8. doi:10.1016/j. autrev.2014.01.031

26. Kim J, Choi SE, Lee KH, Jeong HJ, Shin JI, Lim BJ. Tubulointerstitial Infiltration of M2 Macrophages in Henoch-Schönlein Purpura Nephritis Indicates the Presence of Glomerular Crescents and Bad Clinical Parameters. Biomed Res Int. 2019;2019: 8579619. doi: 10.1155/2019/8579619.

27. Davin JC, Coppo R. Henoch-Schönlein purpura nephritis in children. Nat Rev Nephrol. 2014; 10:563-573. 
28. Vernon MA, Mylonas KJ, Hughes J. Macrophages and renal fibrosis. Semin Nephrol. 2010; 30:302-317.

29. Pillebout E, Jamin A, Ayari H, Housset P, Pierre M, Sauvaget V,et al. Biomarkers of $\operatorname{IgA}$ vasculitis nephritis in children. PLoS One. 2017; 12:e0188718. doi: 10.1371/ journal.pone.0188718.

30. Tan J, Tang Y, Xu Y, Yan S, Xu Y, Tan L, et al. The clinicopathological characteristics of Henoch-Schönlein purpura nephritis with presentation of nephrotic syndrome. Kidney Blood Press Res. 2019;44:754-64. doi: 10.1159/000501459.

31. Li X, Tang M, Yao X, Zhang N, Fan J, Zhou N, et al. A clinicopathological comparison between IgA nephropathy and Henoch-Schönlein purpura nephritis in children: use of the Oxford classification. Clin Exp Nephrol. 2019;23:13821390. doi: 10.1007/s10157-019-01777-8.

32. Trimarchi H, Barratt J, Cattran DC, Cook HT, Coppo R, Haas M, et al. Oxford Classification of IgA nephropathy 2016: an update from the IgA Nephropathy Classification Working Group. Kidney Int. 2017;91:1014-21. doi: 10.1016/j.kint.2017.02.003.

33. Jelusic M, Sestan M, Cimaz R, Ozen S. Different histological classifications for Henoch-Schönlein purpura nephritis: which one should be used? Pediatr Rheumatol. 2019;17:10. Doi: 10.1186/s12969-019-0311-z.

34. Roberts IS, Cook HT, Troyanov S, Alpers CE, Amore A, Barratt J, et al. The Oxford classification of IgA nephropathy: pathology definitions, correlations, and reproducibility. Kidney Int. 2009; 76:546-56. doi:10.1038/ki.2009.168.

35. Sanders JT, Wyatt RJ. IgA nephropathy and HenochSchönlein purpura nephritis. Curr Opin Pediatr. 2008; 20:163-170.

36. Coppo R, Troyanov S, Camilla R, Hogg RJ, Cattran DC, Cook HT, et al. The Oxford IgA nephropathy clinicopathological classification is valid for children as well as adults. Kidney Int. 2010; 77:921-927

37. Xu K, Zhang L, Ding J, Wang S, Su B, Xiao H, et al. Value of the Oxford classification of IgA nephropathy in children with Henoch-Schönlein purpura nephritis. J Nephrol. 2018; 31:279-86. doi: 10.1007/s40620-017-0457-z.

Copyright $(2020$ The Author(s); Published by Nickan Research Institute. This is an open-access article distributed under the terms of the Creative Commons Attribution License (http://creativecommons.org/licenses/by/4.0), which permits unrestricted use, distribution, and reproduction in any medium, provided the original work is properly cited. 